\title{
Suppression of beating noise of narrow-linewidth erbium-doped fiber ring lasers by use of a semiconductor optical amplifier
}

\author{
Lei Xu, Ivan Glesk, Darren Rand, Varghese Baby, and Paul R. Prucnal \\ Department of Electrical Engineering, Princeton University, Princeton, New Jersey 08544
}

Received December 5, 2002

\begin{abstract}
Beating noise in narrow-linewidth erbium-doped fiber ring lasers puts severe limitations on applications of the lasers. We demonstrate the suppression of beating noise in fiber ring lasers by using a semiconductor optical amplifier in the laser cavity, which acts as a high-pass filter. Two different ring structures are presented as examples to demonstrate this beating noise suppression. (C) 2003 Optical Society of America

OCIS codes: $140.3500,060.4510$.
\end{abstract}

Low-noise, narrow-linewidth fiber ring lasers that use erbium-doped fiber amplifiers (EDFAs) as the gain medium have attracted much research interest because of their potential applications such as optical sensors and light sources for optical communications. However, intensity noise from these fiber ring structures can put severe limitations on the lasers' applications. Sources of output intensity noise include beating noise, environmental perturbations, thermal noise, and fluctuations in pump power. Various methods for partially reducing output noise have been proposed. ${ }^{1-3}$ Beating noise, which is generated as a result of beating between the lasing mode and amplified spontaneous emission in the strongly suppressed side modes, causes large fluctuations in the intensity of the laser output. ${ }^{4}$ Efficient suppression of the beating noise is crucial for a fiber laser used as a continuous-wave light source. Sanders et al. reduced beating noise from an erbium-doped fiber laser by intracavity spectral filtering. However, this filtering resulted in a decrease in the output optical power. ${ }^{1}$ Here we demonstrate the suppression of beating noise in narrow-linewidth erbium-doped fiber ring lasers by using a semiconductor optical amplifier (SOA). The SOA acts as a high-pass filter because of its relatively fast carrier recovery rate (several hundred picoseconds to a couple of nanoseconds) and the gain saturation effect. ${ }^{5}$ Inasmuch as the frequency of the beating noise is related to the fundamental longitudinal mode of the laser cavity, and the length of the fiber ring cavity is typically several meters to tens of meters, the noise spectrum is primarily in the low-frequency region. Thus the introduction of a SOA into the laser cavity dramatically suppresses the low-frequency intensity noise of the laser. We demonstrate this suppression for two ring structures: one based on a Lyot filter structure ${ }^{6-8}$ and the other based on a fiber Bragg grating. ${ }^{9}$

The first laser structure in our experiments is shown in Fig. 1. The EDFA (Princeton Optics) has an erbium-doped fiber length of $\sim 10 \mathrm{~m}$. A polarization-maintaining (PM) fiber, a rotating polarizer, and a polarization controller in the cavity form a Lyot filter, which provides filtering and a wavelength- selection mechanism. ${ }^{6}$ Ten percent of the light is coupled out of the laser through an optical coupler. Adjusting the polarization controller allows the laser to work in a state of narrow-linewidth lasing. ${ }^{7}$ When a SOA is not used in the laser cavity, the optical spectrum of the output light is as shown in Fig. 2. The 3 -dB spectral bandwidth is less than $0.01 \mathrm{~nm}$, which is the minimal resolution of the optical spectrum analyzer. By changing the polarization controller and the rotating polarizer we can tune the wavelength of the output light in a $25-\mathrm{nm}$ range. ${ }^{8}$

To look at the intensity noise we convert the laser output signal into an electrical signal through a $20-\mathrm{GHz}$ photodetector (New Focus 1414), and the rf signal is shown with a rf spectrum analyzer (HP70004A). The optical input to the photodetector is kept at $\sim-3 \mathrm{dBm}$. Figures 3 and 4 show the rf spectra of the laser output signal in the ranges $0-6 \mathrm{GHz}$ and $0-100 \mathrm{MHz}$, respectively. When the SOA is not included in the laser cavity, the laser output shows a strong intensity noise [Fig. 3(a)]. From Fig. 4(a) we can see that the noise frequency peaks are a harmonic frequency sequence, and the first one is at $16 \mathrm{MHz}$, which corresponds to a laser cavity length of $\sim 12.5 \mathrm{~m}$. These harmonic frequency components are caused by beating of the lasing mode and the amplified stimulated emission of the strongly suppressed side modes. With the SOA in the laser cavity, we can see that the intensity noise is dramatically suppressed, as is shown in Figs. 3(b) and 4(b). The SOA (Alcatel 1901)

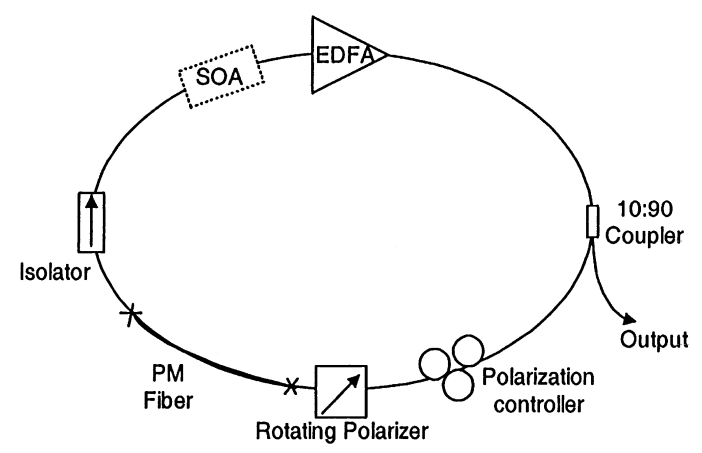

Fig. 1. Experimental setup. 


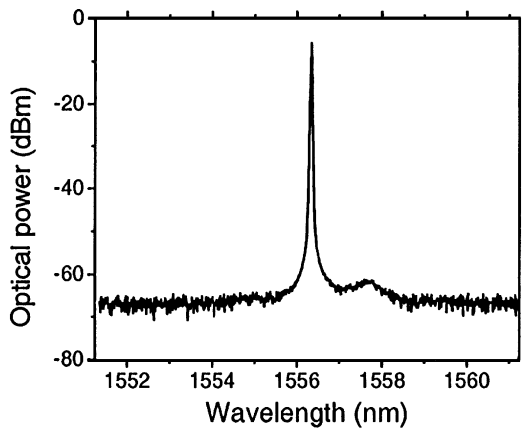

Fig. 2. Output optical spectrum from the laser in Fig. 1 without a SOA.

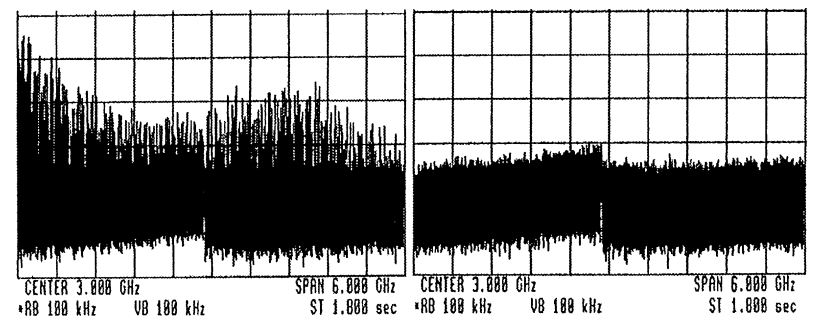

(a)

(b)

Fig. 3. rf spectrum of the light output from the laser in Fig. 1: (a) without and (b) with a SOA in a $0-6-\mathrm{GHz}$ span (vertical scale, $10 \mathrm{~dB} /$ division).

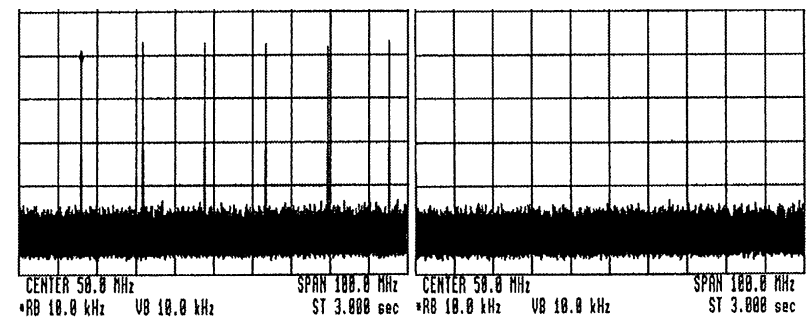

(a)

(b)

Fig. 4. rf spectrum of the light output from the laser in Fig. 1: (a) without and (b) with a SOA in a $0-100-\mathrm{MHz}$ space (vertical scale, $10 \mathrm{~dB} /$ division).

injection current is $81 \mathrm{~mA}$. For comparison with Fig. 2, the output optical spectrum is shown in Fig. 5. Although the optical spectra in Figs. 2 and 5 both have narrow linewidths, their rf spectra can be quite different. Therefore it is important to look at both the optical and the rf spectra to evaluate a narrow-linewidth laser.

We further studied the output power of the laser under several EDFA and SOA currents (Fig. 6). The current of the EDFA refers to the current for the pump diode, and it can be varied from 0 to $190 \mathrm{~mA}$. When there is a small SOA injection current, the SOA acts as an absorbing material, and the output power of the laser is decreased. When the SOA current increases, the increased saturation power and the gain of the SOA result in improved output power. When the EDFA current is increased further, the maximum output power will be limited by the saturation power of the SOA. In our experiments we found that the $\mathrm{SOA}$ injection current has to be larger than $35 \mathrm{~mA}$ for efficient reduction of the intensity noise. When the SOA instead of the EDFA is used solely as the gain medium in the cavity, the laser output does not have noticeable beating noise, and the output power is at -4.9 and $-1.9 \mathrm{dBm}$ for SOA injection currents of 100 and $180 \mathrm{~mA}$, respectively.

To show that the beating noise phenomenon and its suppression by a SOA are independent of the structure of the fiber ring laser, we used another structure, based on a fiber Bragg grating (FBG). ${ }^{9}$ The laser structure is shown in Fig. 7(a). The FBG has a 3-dB bandwidth of $0.3 \mathrm{~nm}$. The optical spectrum of the laser without the SOA is shown in Fig. 7(b). The rf spectra of the laser without and with the SOA are shown in Figs. 8(a) and 8(b), respectively. In Fig. 8(a) the frequency of the first noise spectral peak is at $14 \mathrm{MHz}$. In comparison with the previous laser

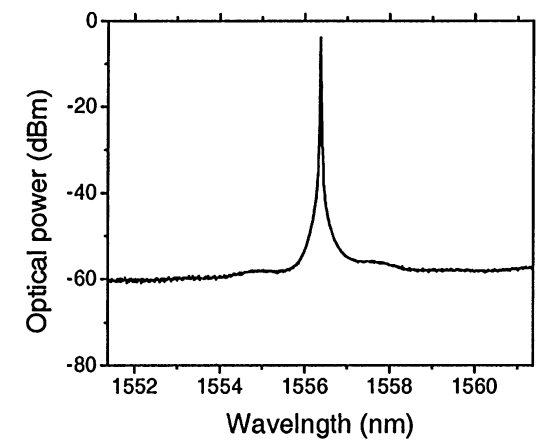

Fig. 5. Output optical spectrum of the laser in Fig. 1 with a SOA.

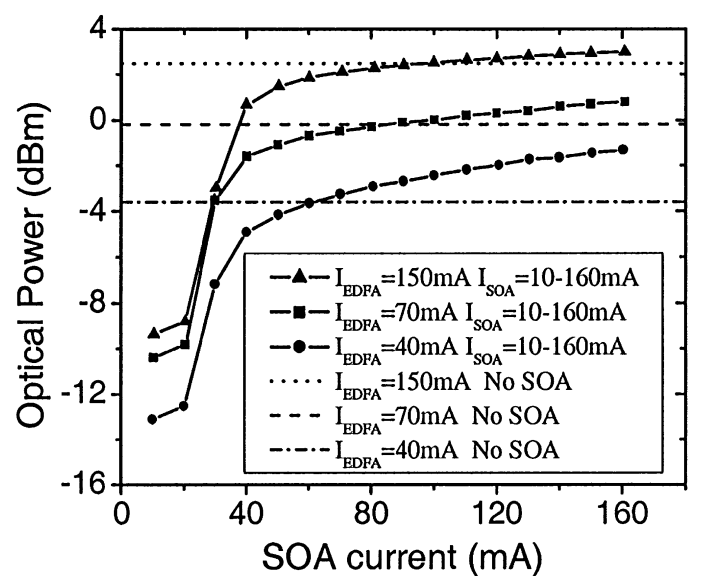

Fig. 6. Optical power of the laser output light under several EDFA and SOA currents.

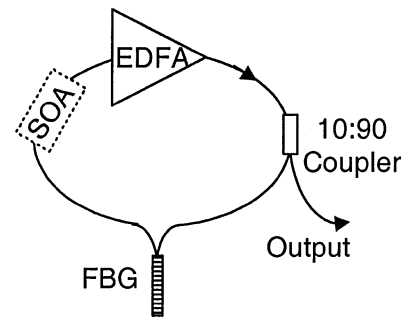

(a)

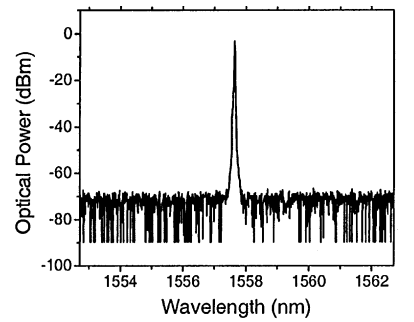

(b)
Fig. 7. (a) FBG-based fiber ring laser and (b) output optical spectrum without a SOA. 


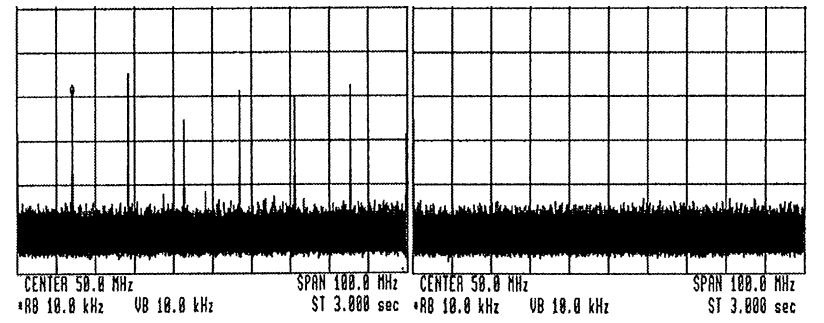

(a)

(b)

Fig. 8. rf spectra from a FBG-based erbium-doped fiber ring laser: (a) without and (b) with a SOA in a 0-100-MHz span (vertical scale, $10 \mathrm{~dB} /$ division).

structure, the period of the harmonic frequency sequence is different and the change corresponds to the difference in the lengths of the two laser cavities. This cavity-length-dependent change in noise spectrum, unlike other noise sources such as pumping fluctuations, is characteristic of beating noise. Figure 8 (b) shows that the beating noise is dramatically suppressed by a SOA with an injection current of $80 \mathrm{~mA}$.

Compared with an EDFA, a SOA has a relatively fast carrier recovery rate and low gain saturation power, resulting in high-pass filter characteristics. In the experimental measurements ${ }^{5}$ the SOA response decreases in the low-frequency region, which is as much as several gigahertz for high input intensity levels. This is enough to suppress the beating noise of an erbium-doped fiber ring laser.

In conclusion, beating noise in erbium-doped fiber ring lasers puts severe limitations on the applications of such lasers. We have demonstrated suppression of beating noise by use of a semiconductor optical amplifier. This SOA acts as a high-pass filter in the laser cavity. Two ring structures have been presented to demonstrate beating noise and its suppression, showing that beating noise is a common consequence of a ring structure that can be suppressed by a SOA.

The authors acknowledge the support of Army Research Office Multiuniversity Research Initiative Prime Grant DAAD 19-00-1-0165. L. Xu's e-mail address is leixu@ee.princeton.edu.

\section{References}

1. S. Sanders, N. Park, J. W. Dawson, and K. J. Vahala, Appl. Phys. Lett. 61, 1889 (1992).

2. Y. Cheng, J. T. Kringlebotn, W. H. Loh, R. I. Laming, and D. N. Payne, Opt. Lett. 20, 875 (1995).

3. G. De Geronimo, S. Taccheo, and P. Laporta, Electron. Lett. 33, 1336 (1997).

4. S. Sanders, J. W. Dawson, N. Park, and K. J. Vahala, Appl. Phys. Lett. 60, 2583 (1992).

5. K. Sato and H. Toba, IEEE J. Sel. Top. Quantum Electron. 7, 328 (2001).

6. D. Zhou, P. R. Prucnal, and I. Glesk, IEEE Photon. Technol. Lett. 10, 781 (1998).

7. L. Xu, B. C. Wang, V. Baby, I. Glesk, and P. R. Prucnal, IEEE Photon. Technol. Lett. 14, 149 (2002).

8. L. Xu, B. C. Wang, V. Baby, I. Glesk, and P. R. Prucnal, in LEOS 2001 (Institute of Electrical and Electronics Engineers, Piscataway, N.J., 2001), Vol. 2, pp. 421-422.

9. G. A. Ball and W. W. Morey, Opt. Lett. 19, 1979 (1994). 